${ }^{9}$ Fielding, D. W. (1975). Personal communication.

10 Jones, K. L., Smith, D. W., Ulleland, C. N., and Streissguth, A. P. (1973). Pattern of malformation in offspring of chronic alcoholic mothers. Lancet, 1, 1267-1271.

11 Jones, K. L., Smith, D. W., Streissguth, A. P., and Myrianthopoulos, N. C. (1974). Outcome of offspring of chronic alcoholic women. Lancet, 1, 1076-1078.

12 Vaisman, A. I. (1967). Work in surgical theatres and its effect on the health of anaesthesiologists (in Russian, English summary). Eksperimental 'naya khirurgiya $i$ Anesteziologiya, 12, 44-49.

13 Kullander, S., and Källén, B. (1976). A prospective study of drugs in pregnancy. I. Psychopharmaca. Acta Obstetrica et Gynaecologica Scandinavica, 55, 25-33.

14 Kullander, S., and Källén, B. (1976). A prospective study of drugs in pregnancy. II. Anti-emetic drugs. Acta Obstetrica et Gynaecologica Scandinavica, 55, 105-111.

15 Kullander, S., and Källén, B. (1976). A prospective study of drugs in pregnancy. III. Hormones. Acta Obstetrica et Gynaecologica Scandinavica, 55, 221-224.

${ }^{16}$ Kullander, S., and Källén, B. (1976). A prospective study of drugs in pregnancy. IV. Miscellaneous drugs. Acta Obstetrica et Gynaecologica Scandinavica, 55, 287-295.

17 Dudgeon, J. A. (1976). Infective causes of human malformations. British Medical Bulletin, 32, 77-83.

${ }^{18}$ Sheppard, S., Smithells, R. W., Peckham, C., Dudgeon, J. A., and Marshall, W. C. (1977). National congenital rubella surveillance 1971-1975. Health Trends, 9, 38-41.

${ }^{19}$ Forrest, J. M., Menser, M. A., and Bergen, J. A. (1971). High frequency of diabetes mellitus in young adults with congenital rubella. Lancet, 2, 332-334.

${ }^{20}$ Hardy, J. B., McCracken, G. H., Gilkeson, M. R., and Sever, J. L. (1969). Adverse fetal outcome following maternal rubella after the first trimester of pregnancy. Journal of the American Medical Association, 207, 24142420.

\section{R. W. SMITHELlS}

Department of Paediatrics and Child Health, University of Leeds, 27 Blundell Street, Leeds LSI 3ET.

\title{
Role of circulating soluble immune complexes in disease
}

A wide variety of chronically disabling diseases are thought to be caused by circulating soluble immune complexes. These include collagen vascular diseases, ${ }^{1}$ rheumatoid arthritis, ${ }^{2}$ glomerulonephritis, ${ }^{3}$ and possibly chronic inflammatory bowel diseases. ${ }^{4}$ Antigenantibody complexes are deposited in the blood vessels of affected organs and initiate damage by activation of the complement system, eliciting an inflammatory response. $^{3}$

The evidence for this damaging role of complexes comes largely from animal studies, in particular those in which chronic glomerulonephritis is induced by repeated antigen administration. ${ }^{5}$ The similarity of immunoglobulin deposition shown by immunofluorescence in human nephritis and in the animal experimental disease suggests that circulating complexes play a role in both. Only a few animals acquire chronic nephritis as a result of repeated antigen dosage, and they respond with predominantly nonprecipitating antibody which is inefficient at antigen elimination. ${ }^{6}$ In man, the rare complement deficiencies, in particular homozygous $\mathrm{C} 1 \mathrm{r}$ and $\mathrm{C} 2$ are associated with immune complex syndromes. ${ }^{7}$ Antibody, complement, and phagocytes are all involved in antigen clearance and a fundamental defect in their function may underlie immune complex disease. However, in the majority of patients with such diseases the immunodeficiency is probably only relative and therefore difficult to detect.
Recently, methods have been developed for the detection of circulating soluble immune complexes in sera and their presence has been confirmed in a variety of diseases. ${ }^{8}$ However, mere detection is insufficient proof of pathogenicity, for it is probable that not all immune complexes are damaging, and sensitive methods detect low levels in healthy individuals. Similarly, raised levels are found in pregnancy which fall after delivery, suggesting that certain types of complex may modulate the immune response in an advantageous manner, thus possibly preventing maternal rejection of that mosaic of foreign antigens, the fetus. ${ }^{9}$ Facilitation or enhancement of malignant tumour growth may also be due to certain types of immune complexes. ${ }^{10}$

Antigens involved in immune complex diseases may be of either exogenous or endogenous origin. Exogenous antigens include administered therapeutic agents producing serum sickness, inhaled and ingested antigens, and microbial and parasitic products derived from invading organisms. In diseases initiated primarily by exogenous antigens, the inflammatory process may release endogenous antigens which perpetuate the disease. Similarly crossreaction of endogenous antigens with antibodies formed towards exogenous antigens may be another mechanism leading to persistent inflammation. This mechanism probably applies in poststreptococcal glomerulonephritis; in systemic lupus erythematosus 
it is possible that the nuclear antigens identified have been induced by cross-reactivity to viral DNA. None the less, in only a few rare instances have specific viruses, bacteria, or protozoa been identified in human immune complex diseases.

It is becoming clear that immune complexes are heterogeneous not only in the antigen involved but also in immunoglobulin class, and complement binding capacity as well as size. The rate of formation is also important, as well as the capacity of the reticuloendothelial system, especially the macrophages of the liver and spleen, for clearing them from the circulation. Little is known of what determines immune complex deposition in a particular organ and why different organs are affected in different diseases. The kidney is probably particularly vulnerable because of its large blood flow and its filtering role, but complement binding complexes may also be preferentially deposited due to the $\mathrm{C} 3$ receptors on the glomerular epithelial cells. ${ }^{11}$

The size of complexes is important since it has been shown experimentally that large complexes are easily cleared by the reticuloendothelial system, whereas small ones are not. ${ }^{12}$ Similarly, smaller complexes of bovine serum albumin with antibody were nephrotoxic in animals whereas larger ones were not. ${ }^{13}$ Such observations have recently been confirmed for human disease in that size variation of complexes may account for the different clinical manifestations of systemic lupus erythematosus; those patients with renal signs had predominantly medium sized IgG complexes, whereas extrarenal manifestations were associated with very large IgG complexes. ${ }^{14}$

It is not surprising that such variation exists, and it therefore becomes clear why few of the many methods developed for immune complex detection correlate with each other. The techniques may depend on physicochemical properties of the complexes (cryoprecipitation or polyethylene glycol precipitation), on recognition proteins such as $\mathrm{Clq}$ or antiglobulins, or on interaction of cells which bind complexes by $\mathrm{C} 3 \mathrm{~b}$ or $\mathrm{Fc}$ receptors (Raji cells, macrophages, and B lymphocytes). ${ }^{15}$ None of the published methods are capable yet of characterising all the constituents of complexes, but the knowledge of the capabilities and limitations of individual methods enables useful information to be obtained by using more than one method to analyse sera.

Good correlation has been observed between clinical activity and circulating immune complexes in a variety of diseases. Systemic lupus erythematosus (SLE) is an example of a chronic allergic disorder in which the activity of the disease appears to correlate well with levels of complexes. ${ }^{14}$ Although the complexes are probably not the only cause of damage in SLE, they are responsible for the nephritis which commonly kills the patient. Monitoring patients with lupus nephritis on immunosuppression is difficult as other immunological parameters such as C3 or DNA binding do not correlate well with disease activity. ${ }^{16}$ Estimation of circulating soluble immune complexes may prove to be a sensitive indication of when to start or discontinue treatments such as plasmapheresis or methyl prednisolone pulse therapy. It is possible that the treatment of diseases such as juvenile chronic polyarthritis may benefit from similar monitoring. In chronic infections such as endocarditis, the associated arthritis, nephritis, splenomegaly, and thrombocytopenia probably result from circulating immune complexes and higher levels are detected in patients with extravalvular lesions than in those without. ${ }^{17}$ In individuals in whom no organism is identified on blood culture, the disappearance of circulating immune complexes should prove useful in monitoring antibiotic therapy.

At present most methods only detect complexes which contain IgG and bind complement. Yet, other immunoglobulin classes may be involved in immune complex formation. Henoch-Schönlein purpura has all the hallmarks of an immune complex disease with arthritis, nephritis, and purpuric skin rashes. Both skin and kidney lesions demonstrate IgA deposition by immunofluorescence. Cryoglobulins have been found in patients with active disease $\mathrm{e}^{18}$ and although circulating immune complexes of either IgG or complement binding variety are not usually found, specific IgA-containing complexes may be demonstrated. ${ }^{19}$ It is not known why IgA complexes are associated with this disease but similar mechanisms may be involved in the recurrent haematuria of childhood syndrome (mesangial IgA/IgG deposition) in which IgA complexes are also found (J. F. Mowbray, personal communication).

The presence of circulating immune complexes was expected in the diseases described above, but not in steroid-responsive nephrotic syndrome. Evidence for the immunologically-mediated cause of this disease is circumstantial, despite successful treatment with immunosuppressive drugs. There is often a history of atopy and patients occasionally relapse after polleninduced hayfever. ${ }^{20}$ Sera from patients in relapse contain raised levels of immunoconglutinin, ${ }^{21}$ an autoantibody to activated C3, and such sera also inhibit complement-dependent rosette formation by B lymphocytes. ${ }^{22}$

These two observations suggest that complement is being activated in vivo. Although immunoglobulin deposition cannot be detected in kidney biopsy specimens by immunofluorescence, raised levels of IgG-containing immune complexes are found in the sera of patients in relapse. ${ }^{23}$ These complexes are not due to the nephrotic state since they do not occur in 
the congenital nephrotic syndrome. The complexes contain IgG but do not bind C1q, suggesting that complement is activated by the alternate pathway. That such large complexes $\left(2-2 \cdot 5 \times 10^{6} \mathrm{MW}\right)$ do not become deposited in the glomerulus is surprising, but it is possible that they cause proteinuria either by activation of a lymphokine system ${ }^{24}$ or by altering the charge on the glomerular basement membrane. Alternatively, it is just as possible that they are an epiphenomenon of the agent provoking the disease.

The finding of circulating immune complexes in sera of patients with a particular disease does not necessarily mean that the complexes are the pathogenetic cause. Although in many instances good correlation with disease activity is obtained, raised levels of complexes may be secondary events. In chronic inflammatory bowel disease it is likely that the diseased gastrointestinal mucosa allows increased antigen entry into the circulation, thereby leading to antigen-antibody complex formation. These complexes are unlikely to be initial provoking causes, but may be responsible for the extragastrointestinal symptoms such as arthritis and uveitis. Similarly, in asthma, the late reaction leading to status asthmaticus may result from immune complexes becoming localised in the lung due to increased vascular permeability after the histamine release of the classical type I allergic reaction. Such localisation of nonspecific complexes by specific IgE antibody responses may occur in other diseases since in experimental serum sickness there is evidence of basophil depletion and mast cell degranulation occurring at the time of immune complex deposition. 25

Future developments in immune complex technology must be capable not only of detecting the complexes, but of characterising their antigens and other constituents, as well as their size. We now need to identify diseases associated with immune complexes containing defined antigens and to investigate the effect of immune complex removal, antigen avoidance, and hyposensitisation on the complexes and on the disease state. Chronic immune complex disease is thought to be a result of poor macrophage function and antibody response, so techniques of enhancing the immune response may be beneficial. Artificial removal of complexes by plasmapheresis or by specific immunoabsorbent columns may well benefit patients during acute exacerbations. In children it is possible that such treatments may result in sustained remission, as it may permit maturation of a relatively defective immune response.

\section{References}

${ }^{1}$ Agnello, V., Koffler, D., Eisenberg, J. W., Winchester, R. J., and Kunkel, H. G. (1971). Clq precipitins in the sera of patients with systemic lupus erythematosus and other hypocomplementemic states; characterization of high and low molecular weight types. Journal of Experimental Medicine, 134, 228S-241S.

2 Zubler, R. H., Nydegger, V., Perrin, L. H., Fehr, K., McCormick, J., Lambert, P. H., and Miescher, P. A. (1976). Circulating and intra-articular immune complexes in patients with rheumatoid arthritis. Journal of Clinical Investigation, 57, 1308-1319.

${ }^{3}$ Cochrane, C. G., and Koffler, D. (1973). Immune complex disease in experimental animals and man. Advances in Immunology, 16, 185-264.

4 Jewell, D. P., and MacLennan, I. C. M. (1973). Circulating immune complexes in inflammatory bowel disease. Clinical and Experimental Immunology, 14, 219-226.

${ }^{5}$ Unanue, E. R., and Dixon, F. J. (1967). Experimental glomerulonephritis: immunological events and pathogenetic mechanisms. Advances in Immunology, 6, 1-90.

${ }^{6}$ Pincus, T., Haberkern, R., and Christian, C. L. (1968). Experimental chronic glomerulitis. Journal of Experimental Medicine, 127, 819-831.

7 Alpers, C. A., and Rosen, F. S. (1974). The role of complement in vivo as revealed by genetic defects. Progress in Immunology, II., Vol. I, pp. 201-206. Ed. by L. Brent and J. Holborow. North-Holland, Amsterdam.

${ }^{8}$ WHO Scientific Group (1977). The Role of Immune Complexes in Disease. Technical Report Series 606. World Health Organisation, Geneva.

${ }^{9}$ Masson, P. L., Delire, M., and Cambiaso, C. L. (1977). Circulating immune complexes in normal human pregnancy. Nature, 266, 542-543.

${ }^{10}$ Hellström, K. E., and Hellström, I. (1974). Lymphocytemediated cytotoxicity and blocking serum activity to tumour antigens. Advances in Immunology, 18, 209-277.

11 Gelfand, M. C., Frank, M. M., and Green, I. (1975). A receptor for the 3 rd component of complement in the human renal glomerulus. Journal of Experimental Medicine, 142, 1029-1034.

12 Mannik, M., and Arend, W. P. (1971). Fate of preformed immune complexes in rabbits and rhesus monkeys. Journal of Experimental Medicine, 134, 19-31S.

13 Germuth, F. G. Jr., Senterfit, L. B., and Dreesman, G. R. (1972). Immune complex disease. V. The nature of the circulating complexes associated with glomerular alterations in the chronic BSA-rabbit system. Johns Hopkins Medical Journal, 130, 344-357.

${ }^{14}$ Levinsky, R. J., Cameron, J. S., and Soothill, J. F. (1977). Serum immune complexes and disease activity in lupus nephritis. Lancet, 1, 564-567.

15 Soothill, J. F. (1977). Chairman's summary. Detection and measurement of circulating soluble antigen-antibody complexes. Annals of the Rheumatic Diseases, 36, Suppl. 1, 64-66.

${ }^{16}$ Cameron, J. S., Lessof, M. H., Ogg, C. S., Williams, B. D., and Williams, D. G. (1976). Disease activity in the nephritis of systemic lupus erythematosus in relation to serum complement concentrations. Clinical and Experimental Immunology, 25, 418-427.

17 Bayer, A. S., Theofilopoulous, A. N., Eisenberg, R., Dixon, F. J., and Guze, L. B. (1976). Circulating immune complexes in infective endocarditis. New England Journal of Medicine, 295, 1500-1505.

${ }^{18}$ Garcia-Fuentes, M., Chantler, C., and Williams, D. G. (1977). Cryoglobulinaemia in Henoch-Schönlein purpura. British Medical Journal, 2, 163-165.

${ }^{19}$ Levinsky, R. J., and Soothill, J. F. (1977). A test for antigen-antibody complexes in human sera using IgM of rabbit antisera to human immunoglobulins. Clinical and Experimental Immunology, 29, 428-435. 
${ }^{20}$ Hardwicke, J., Soothill, J. F., Squire, J. F., and Holti, G. (1959). Nephrotic syndrome with pollen hypersensitivity. Lancet, 1, 500-502.

21 Ngu, J. L., Barratt, J. M., and Soothill, J. F. (1970). Immunoconglutinin and complement changes in steroid sensitive relapsing nephrotic syndrome of children. Clinical and Experimental Immunology, 6, 109-116.

${ }^{22}$ Smith, M. D., Barratt, T. M., Hayward, A. R., and Soothill, J. F. (1975). The inhibition of complement dependent lymphocyte rosette formation by the sera of children with steroid sensitive nephrotic syndrome. Clinical and Experimental Immunology, 21, 236-243.

${ }^{23}$ Levinsky, R. J., Malleson, P. N., Barratt, T. M., and Soothill, J. F. (1978). Circulating immune complexes in steroid responsive nephrotic syndrome. New England Journal of Medicine, 298, 126-129.
24 Lagrue, G., Xheneumont, S., Branellec, A., Hirbic, G., and Weil, B. (1975). A vascular permeability factor elaborated from lymphocytes. 1. Demonstration in patients with nephrotic syndrome. Biomedicine, 23, 37-40.

25 Benveniste, J., Egido, J., and Gutierrez-Millet, V. (1976). Evidence for the involvement of the IgE-basophil system in acute serum sickness of rabbits. Clinical and Experimental Immunology, 26, 449-456.

ROLAND J. LEVINSKY

Department of Immunology, Institute of Child Health, 30 Guilford Street, London WCIN IEH. 